\title{
Alternate Approaches to Coping in Latinx Adolescents From Immigrant Families
}

By: Laura M. Gonzalez, Yesenia Mejia, Andrea Kulish, Gabriela Livas Stein, Lisa Kiang, Deja Fitzgerald, and Alyson Cavanaugh

Gonzalez, L. M., Mejia, Y., Kulish, A., Stein, G. L., Kiang, L., Fitzgerald, D., \& Cavanaugh, A. (2020). Alternate Approaches to Coping in Latinx Adolescents From Immigrant Families. Journal of Adolescent Research. https://doi.org/10.1177/0743558420914083

\section{***;(C) 2020 The Authors. Reprinted with permission. No further reproduction is authorized without written permission from SAGE Publications. This version of the document is not the version of record. Reuse of this document is restricted to non-commercial and no derivative uses. $* * *$}

\begin{abstract}
:
The aims of this mixed-methods study were (a) to explore quantitatively the fit of the COPE inventory (Coping Orientation to Problems Experienced) for Latinx youth from immigrant families, and (b) to explore qualitatively aspects of coping in this population. Participants were 175 Latinx adolescents (51\% female), most of whom were U.S.-born with immigrant parents $(88 \%)$ and primarily of Mexican origin (89\%). The average age was 12.9 years for the quantitative study and 15.7 years for the qualitative study. Qualitative interviews engaged a subset $(n=14)$ of the full study. All participants lived in the southeastern United States and the research received institutional review board (IRB) approval. The confirmatory factor analysis of the COPE inventory was not a good fit for the sample. Thus, an exploration of alternative approaches to coping was undertaken (exploratory factor analysis [EFA] and qualitative interviews). A three-factor solution was selected as the best fit in the EFA; the researchers labeled the factors as "purposeful cognitive/behavioral engagement," "support seeking," and "separation/disengagement." In the qualitative interview data, five main themes were described (relational coping, positive thinking/self-talk, planning, separating/disengaging, and behavioral coping). The researchers suggest implications for reframing coping with Latinx participants or collectivist groups, emphasizing the central role of cultural values.
\end{abstract}

Keywords: coping | Latinx immigrants | exploratory factor analysis | collectivism | qualitative

\section{Article:}

Coping research bridges the conceptual and the practical worlds of psychology in that it is "distinguished by its focus on what children actually do in dealing with specific difficulties in real-life contexts" (Skinner \& Zimmer-Gembeck, 2007, p. 120). Thus, understanding the coping strategies that children employ is valuable because it can help psychologists and mental health professionals improve the effectiveness of those efforts. Particularly in youth and adolescence, improved coping strategies can bolster the ability to adapt, regulate, and overcome normative developmental challenges and unanticipated stressors (McDermott, Umaña-Taylor, \& Zeiders, 2019). 


\section{Centering Culture in Coping Definitions}

One gap that is slowly being addressed in the coping literature is a more nuanced understanding of how coping may look in varied cultural communities, families, and individuals (Greer, 2007; Kuo, 2011; Yeh et al., 2006). Most of the baseline coping research emerged from a whitestream framework wherein coping is an individual activity (Kuo, 2013). Thus, researchers may need to consider that coping in populations with collectivistic cultural values could have different markers or emphases (Hernández \& Villodas, 2019; Kuo, 2013; Moore \& Constantine, 2005; Yeh et al., 2006). In a collectivist cultural orientation, family or close relationships are emphasized over individual needs (Moore \& Constantine, 2005). Maintaining harmony in relationships and functioning in an interdependent manner are key tenets (Yeh et al., 2006). A collective worldview may predispose some individuals to seek social support as a primary coping strategy, while also underscoring the need for forbearance in some situations (the desire to minimize or hide problems so as not to burden others or disrupt key relationships; Moore \& Constantine, 2005). Thus, coping might need to be reframed from an interdependent point of view (embedded in family or group), and not an individual point of view (coping through individual efforts), to be relevant to collective cultures such as immigrants from Latin America (Kuo, 2013; Moore \& Constantine, 2005).

In addition to collectivism, familism is another key cultural value that could have relevance for coping in Latinx communities (Kuo, 2013; Stein et al., 2014). Familism includes deriving a sense of meaning from being a valued part of the family system (e.g., feeling obliged to serve the family in a specific role) as well as a sense of loyalty and support between family members. Coping framed from a Latinx point of view should consider this value for support, cohesion, loyalty, and self-subjugating behavior (Kuo, 2013; Santiago et al., 2016), which is not central to most current measures or definitions.

Finally, the existence of pervasive, uncontrolled stressors commonly experienced in minoritized communities may not have been contemplated in the foundational coping literature (McDermott, Umaña-Taylor, \& Zeiders, 2019). Thus, given key cultural values that can serve as strengths (collectivism and familism) and the presence of culturally specific stressors (discrimination), it is reasonable to ask whether existing conceptualizations or measures of coping are fitting for Latinx youth from immigrant families (Kuo, 2013). Most quantitative studies that have included Latinx participants have utilized such measures without centering the role of culture and thus interrogating the foundational definition of the construct. Existing studies also do not explore the possibility that cultural strengths or values might contribute to coping in unique, understudied ways that are not captured by current measures.

Indeed, there are disagreements expressed within the coping literature about the best way to conceptualize the construct in general (Connor-Smith et al., 2000). Some of the major distinctions drawn in the existing literature are between problem-focused coping, which uses cognitive techniques to modify the source of stress, and emotion-focused coping, which attempts to reduce the affective impact of the stress (Skinner \& Zimmer-Gemback, 2007). Together, problem-focused and emotion-focused coping have been characterized as engaged coping (proactively trying to address the stressor through emotional support seeking, problem-solving 
behaviors, or cognitive reframing), as compared with a disengaged style, relying on avoidance, denial, and withdrawal (Sanchez et al., 2018).

There are a variety of self-report measures of coping that are represented in the literature; most utilize the framework of engaged and disengaged coping, sometimes called active and avoidance coping (Aldridge \& Roesch, 2008). The item phrasing and underlying assumptions of many of these measures underscore the idea that individuals must discern whether to engage with their stressors (cognitively, emotionally, or behaviorally) or whether they should disengage from the stressor. However, even the coping measures that have been used with members of the Latinx community (Taylor et al., 2018; Umaña-Taylor et al., 2008) have rarely been evaluated for goodness of fit (see McDermott, Umaña-Taylor, \& Martinez-Fuentes, 2019, for an exception). Very few published research studies could be found that actively questioned the underlying assumption that existing frameworks are culturally congruent with the stressors and coping responses of Latinx youth from immigrant families (McDermott, Umaña-Taylor, \& Zeiders, 2019; Santiago et al., 2016).

\section{Dimensions of Stress and Coping in Latinx Immigrant Families}

For immigrants, the host country may offer stressors that are different from what families are primed to expect in their home countries, such as racial/ethnic discrimination, new cultural norms for relationships and behavior, language gaps, and so on (McDermott, Umaña-Taylor, \& Martinez-Fuentes, 2019). Even the process of acculturation can cause stress, as parents and children typically adjust to the host country at differing rates and may start to have conflict about roles and expectations (Brittian et al., 2013). Culturally specific stressors for youth (e.g., discrimination and acculturative stress) are likely to be present in addition to culturally universal stressors (e.g., academic, social, and economic), which make studying how Latinx youth (and other marginalized groups) cope with stress an urgent need (Stein, Gonzalez, \& Huq, 2012; Brietzke \& Perreira, 2017; Brittian et al., 2013). The current sociopolitical climate for immigrants has only increased experiences of stress according to academic studies (Stafford et al., 2019).

McDermott, Umaña-Taylor, and Martinez-Fuentes (2019) utilized the Discrimination Coping Strategies Scale to investigate the potential existence of unique profiles in coping with racialethnic discrimination as a stressor. The researchers postulated three profiles, including Confrontative, Proactive, and Passive/Moderately Proud. In comparison with earlier coping studies with predominantly White participants, McDermott et al. illuminated the fact that strategies for coping with a chronic, systemic, and uncontrollable stressor-like discrimination may not be fully captured with terms like "engaged" or "disengaged." Santiago and colleagues (2016) identified some key culturally relevant variables (familism and ethnic identity exploration) that were associated with greater use of engagement coping strategies among Latinx youth.

In addition, some studies have examined stress and coping in Latinx college students (Gloria et al., 2017; Hernández \& Villodas, 2019; Sanchez et al., 2018) with a focus on engaged/disengaged forms of coping, spiritual coping mechanisms, the influence of genderbased beliefs (i.e., marianismo) on coping, and coping with bicultural stress and racial-ethnic 
microaggressions. Disengaged coping strategies were not associated with positive outcomes in the quantitative studies, with links to negative mental health indicators and lower college persistence attitudes. The only qualitative studies (Brietzke \& Perreira, 2017; Gloria et al., 2017) highlighted the importance of spirituality in the academic coping strategies of Latinx males and a sense of ethnic identity and belonging to one's family, as positive resources for coping. These studies stand out for bringing the culturally influenced perspectives of Latinx youth to bear on possible coping strategies, with the potential to reshape or redefine those constructs. Yet, despite emerging work that has begun to question a "one size fits all" approach, research has yet to systematically interrogate the applicability of prevalent coping models for Latinx youth.

\section{The Current Study}

Thus, the goal of the current study is to step back from the assumption that current models of coping are adequate to capture all aspects of the construct with Latinx participants. The COPE (Coping Orientation to Problems Experienced; Carver et al., 1989) was the measure selected for analysis because "the multi- dimensional design of the COPE is a potential strength in capturing complexities involved in coping processes," (Greer, 2007, p. 261). In other words, the COPE does not result in one overall coping score but is recommended for flexible use in examining the patterns of coping. It has also been validated in studies with adolescents, including some with Latinx youth (e.g., Aldridge \& Roesch, 2008). The two main research questions guiding the study were as follows:

Research Question 1: Does the COPE model provide a good fit for a population of Latinx youth from immigrant households?

Research Question 2: If not, what might coping look like in this population if culturally influenced factors could be included?

Thus, the first step in the current mixed-methods study will be to test the traditional COPE factor structure with confirmatory factor analysis (CFA) and, if the model is excluded, to explore an alternate, culturally informed model of coping in Latinx youth concurrently with qualitative and quantitative data (Hanson et al., 2005). The research team hypothesized that the CFA would not be a good fit for our data, and that we would see a factor emerge with more emphasis on collective or relational coping strategies in our analyses.

\section{Method}

Quantitative Phase

Participants. Participants for the quantitative portion of this study included 175 Latinx adolescents (51\% female) recruited with their mothers as part of a larger data collection project. The majority of the adolescent participants were born in the mainland United States $(88 \%)$ and are primarily of Mexican origin (89\%). Of those not born in the mainland United States, $84 \%$ reported immigrating at or before the age of 10 years. The average household income was US\$23,020 and 59.5\% of the mothers endorsed being married. Educationally, the majority of 
mothers $(45.1 \%)$ reported completing the equivalent of eighth grade or less. The average adolescent age was 12.9 years at the time of quantitative data collection.

Recruitment. According to institutional review board (IRB) approved procedures, adolescents were recruited from contact lists of Latinx students $(n=597)$ provided by two middle schools in semirural North Carolina. Families were contacted principally through phone (up to five attempts) and then by door-to-door procedures for families that were unable to be reached by phone. Eligibility criteria included that the adolescent's biological parents endorsed a Latinx background, that one adolescent from each family could participate, and that the adolescent's mother lived in the home with the adolescent. Once contacted $(n=364)$, a trained research assistant provided the adolescent's mother with information about the study, screened for eligibility, and obtained initial verbal interest from both the mother and the adolescent. Eligible and interested families were then scheduled for a time to complete the surveys in-home. In families with two or more eligible adolescents, the mother chose which one would participate. Verbal consent for the adolescent to participate was obtained from the mother in person, and the adolescent was also asked for assent. A total of 176 families (48\% of those we attempted to contact) completed the surveys; one adolescent was later determined to be ineligible, bringing the final sample size to 175 .

Procedures. Two trained research assistants administered the surveys in the adolescent's home. The adolescents completed the surveys using a laptop with a completion time between 1.5 and 2 hours. All but two of the adolescents chose to participate in English. After completion of the survey, the adolescent received a US\$10 gift card and the mother received a US\$20 gift card.

Measure. The COPE (Carver et al., 1989) was the only measure utilized for the factor analysis. The original COPE (60 items with a Likert-type scale from 1 [don't do this at all] to 4 [do this a $l o t]$ ) included 15 subscales that were associated with three latent factors. The first factor consisted of problem-focused coping and included five subscales: planning, suppression of competing activities, restraint, seeking of instrumental social support, and active coping. The second factor consisted of emotion-focused coping and included six subscales: seeking of emotional social support, positive reinterpretation, acceptance, denial, humor, and religion. The third factor consisted of attempts at disengaging from problems and included four subscales: venting of emotions, behavioral disengagement, mental disengagement, and use of alcohol. Cronbach's alpha scores were between .62 and .92 for all subscales in the original study except mental disengagement ( $\alpha=.45$; Carver et al., 1989).

Data analysis. The Mplus Version 8.1, a CFA employing the maximum likelihood estimation method, was used to compare the original latent COPE structure and evaluate whether it was a good fit in the current Latinx adolescent sample. Several indices were examined to test the overall fit of the model. The comparative fit index (CFI), standardized root mean residual (SRMR), and root mean square error of approximation (RMSEA) were examined. Ideally, the CFI should be close to .95 , and the SRMR and RMSEA should be .08 or lower for a model to have acceptable fit (Hu \& Bentler, 1999). These values were used as guidelines to avoid overgeneralizing (Marsh et al., 2004). 
Subsequent to the CFA, an exploratory factor analysis (EFA) was conducted to examine whether an alternate latent structure provided a better fit to the data. A Geomin rotation was employed because correlated factors were expected and because it is preferred when there is no clear expectation about the true loading structure (Asparouhov \& Muthén, 2009). Several guidelines were used for deciding on the appropriate number of factors to retain. First, the scree plot of the eigenvalues of the variables' correlation matrix was examined. Then, fit indices across three models were compared, for which a range of one to three factors were extracted (models did not converge with additional factors). The same fit indices from the CFA were used to examine the overall fit of these models. Of note, chi-square values were not used as fit indices as these values are a function of sample size and less useful in determining model fit (Bentler \& Bonett, 1980).

Qualitative Phase

Participants. Participants were 14 Latinx adolescents (50\% female) from the larger quantitative sample. All were born in the United States and all were of Mexican origin except one (Nicaraguan origin). The average household income was US\$18,392, and 50\% of the mothers of the adolescent participants endorsed being married. Educationally, the majority of mothers $(42.9 \%)$ reported completing the equivalent of eighth grade or less. The average adolescent age at the time of the qualitative study (1-2 years after the quantitative study) was 15.7 years.

Recruitment. Adolescent participants who were recruited from the full sample $(N=175)$ had indicated interest in being part of future research studies $(n=147)$. Due to the passage of time, many families were unable to be contacted (e.g., disconnected phones and changed numbers). Of the remaining adolescents $(n=25)$, the first 14 families who were eligible and interested (per IRB approval for a sample of 14 participants) were then scheduled for an in-home interview.

Procedures. Per IRB procedures, parents provided consent and adolescents assented to participation. The interview with the adolescents took place at home and was conducted by a clinical psychology graduate student. Interviews were recorded in English, per the adolescents' preference and typically lasted 45 minutes. Participants were provided a US\$20 gift card in appreciation for their time and were given resources related to higher education opportunities.

Qualitative interview. The interview protocol (available upon request) was created by several members of the research team and implemented by one graduate student in order to increase consistency. Sample questions included "Think about a difficult situation you have encountered at school. What do you say, think, or do to get through it? Would you try something different if the situation was at home?" and "Describe a time when you have coped really well with a situation. What worked well for you?" Participants were asked follow-up questions for clarity or further reflection.

Data analysis. Research case numbers were assigned to each interview and names were removed. The audio recordings were transcribed and double-checked by undergraduate researchers supervised by the principal investigators. The graduate student interviewer (third author) led the coding process (under the guidance of the first author) with two advanced undergraduate students who were instructed in thematic analysis (Braun \& Clarke, 2006). The goal of the coding was "to provide a more detailed and nuanced account of one particular theme, 
or group of themes, within the data" (Braun \& Clarke, 2006, p. 11). As the research questions focused on specific situations that required coping skills and youth responses, the semantic approach to coding was most appropriate. The coding team met once a week to review transcripts, identify initial codes, and then moved into the process of grouping those codes into emerging secondary themes and generating labels for the themes. The first author served as auditor in order to enhance trustworthiness. She reviewed the initial codes and emergent themes and asked the coding group clarifying questions (e.g., "Can you provide more details about what you mean by 'talk it out'?"). After a few rounds, the auditor and the coding group agreed on five main labels that captured the majority of the content (see Table 3).

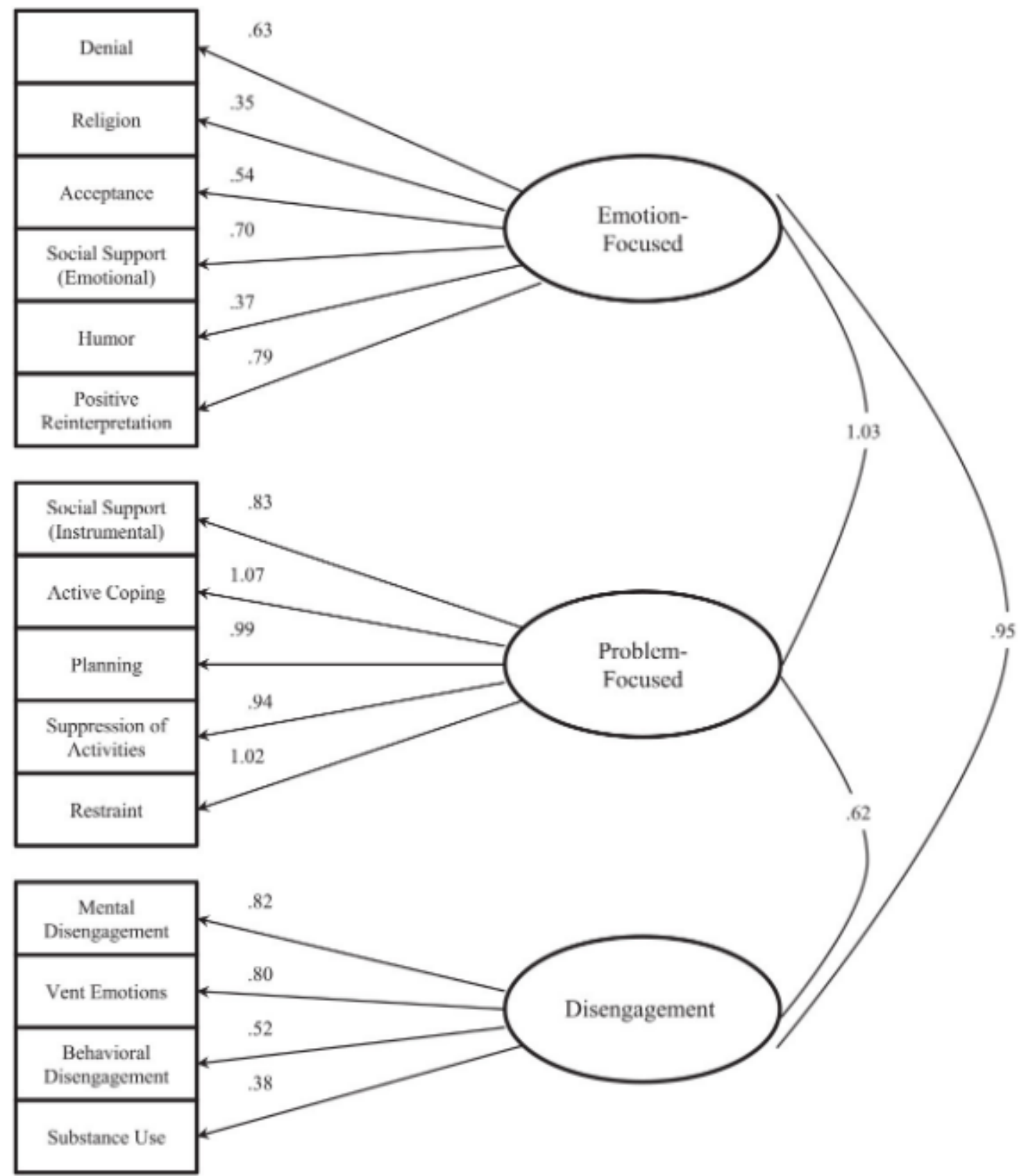

Figure 1. Original latent model for COPE subscales and factor loadings.

Note. Latent constructs are shown in ellipses and indicator variables are shown in rectangles. COPE $=$ Coping Orientation to Problems Experienced. 


\section{Results}

Quantitative Phase

Confirmatory factor analyses revealed that the original COPE three-factor model yielded a poor fit to the data, $\chi^{2}(1692, N=174)=3,004.099, p<.001 ; \mathrm{RMSEA}=0.067$, confidence interval $(\mathrm{CI})=[.063, .071] ; \mathrm{CFI}=.675 ; \mathrm{SRMR}=.099$. The original latent COPE structure along with factor loadings is displayed in Figure 1. The EFA was undertaken next, entering the $15 \mathrm{COPE}$ subscales as units due to the sample size (which did not support entering all 60 items individually). Fit indices for the three identified EFAs are reported in Table 1. The three-factor solution had the best fit indices, $\mathrm{RMSEA}=0.053, \mathrm{CI}=[.028, .074]$; $\mathrm{CFI}=.968$; $\mathrm{SRMR}=.036$. The scree plot also pointed to a three-factor solution; thus, this model was retained. The alternate latent structure for this model along with factor loadings is displayed in Figure 2. The first factor included the subscales of positive reinterpretation, active coping, religion, humor, restraint, acceptance, suppression of activities, and planning. The second factor included the subscales of venting, seeking of instrumental social support, and emotional support. The third factor included mental disengagement, denial, behavioral disengagement, and use of alcohol (see Table 2). Each factor was distinct and only Factors 2 and 3 were significantly correlated $(r=.396)$.

Table 1. Exploratory Factor Analysis (EFA) of Current Study: Comparing Fit Indices Across Three-Factor Solutions.

\begin{tabular}{|lccccccc|}
\hline Factor solution & $\chi^{\mathbf{2}}$ & CFI & TLI & RMSEA & SRMR & AIC & BIC \\
\hline EFA & & & & & & & \\
One-factor & 292.748 & .788 & .753 & .114 & .081 & $4,603.251$ & $4,745.408$ \\
Two-factor & 168.161 & .904 & .867 & .083 & .058 & $4,506.664$ & $4,693.048$ \\
Three-factor & 93.430 & .968 & .947 & .053 & .036 & $4,457.933$ & $4,685.385$ \\
\hline
\end{tabular}

Note. The best fitting model was the three-factor solution indicated by well-defined factors, acceptable model fit, and the scree plot. Standardized factor loadings for this three-factor solution are displayed in Table 2. CFI = comparative fit index; TLI $=$ Tucker-Lewis index; RMSEA $=$ root mean squared error of approximation; SRMR = standardized root mean square residual; $\mathrm{AIC}=$ Akaike information criterion; $\mathrm{BIC}=$ Bayesian information criterion.

Table 2. Exploratory Factor Analysis (EFA) Results of Three-Factor Solution in Current Study.

\begin{tabular}{|c|c|c|c|}
\hline \multirow[b]{2}{*}{ COPE subscales } & \multicolumn{3}{|c|}{ Standardized factor loadings } \\
\hline & 1 & 2 & 3 \\
\hline Positive reinterpretation & $.716^{*}$ & .165 & .058 \\
\hline Active coping & $.700 *$ & $.230^{*}$ & -.002 \\
\hline Religion & $.327 *$ & .135 & .116 \\
\hline Humor & $.298 *$ & -.190 & $.279 *$ \\
\hline Restraint & $.587 *$ & -.019 & $.320 *$ \\
\hline Acceptance & $.555 *$ & .098 & -.026 \\
\hline Suppression of activities & $.713 *$ & -0.13 & .169 \\
\hline Planning & $.736 *$ & $.251^{*}$ & -.028 \\
\hline Venting & -.008 & $.564 *$ & .362 \\
\hline Social support (instrumental) & $.378^{*}$ & $.653 *$ & -.003 \\
\hline Social support (emotional) & .179 & $.796 *$ & .015 \\
\hline Mental disengagement & $.272 *$ & .155 & $.333 *$ \\
\hline Denial & .139 & .091 & $.585 *$ \\
\hline Behavioral disengagement & -.038 & .014 & $.663 *$ \\
\hline Substance use & -.006 & -.019 & $.456 *$ \\
\hline
\end{tabular}

Note. Items with bold font loadings are those whose factor explains at least $15 \%$ of the indicator's variance. COPE $=$ Coping Orientation to Problems Experienced. ${ }^{*} \mathrm{p}<.05$. 


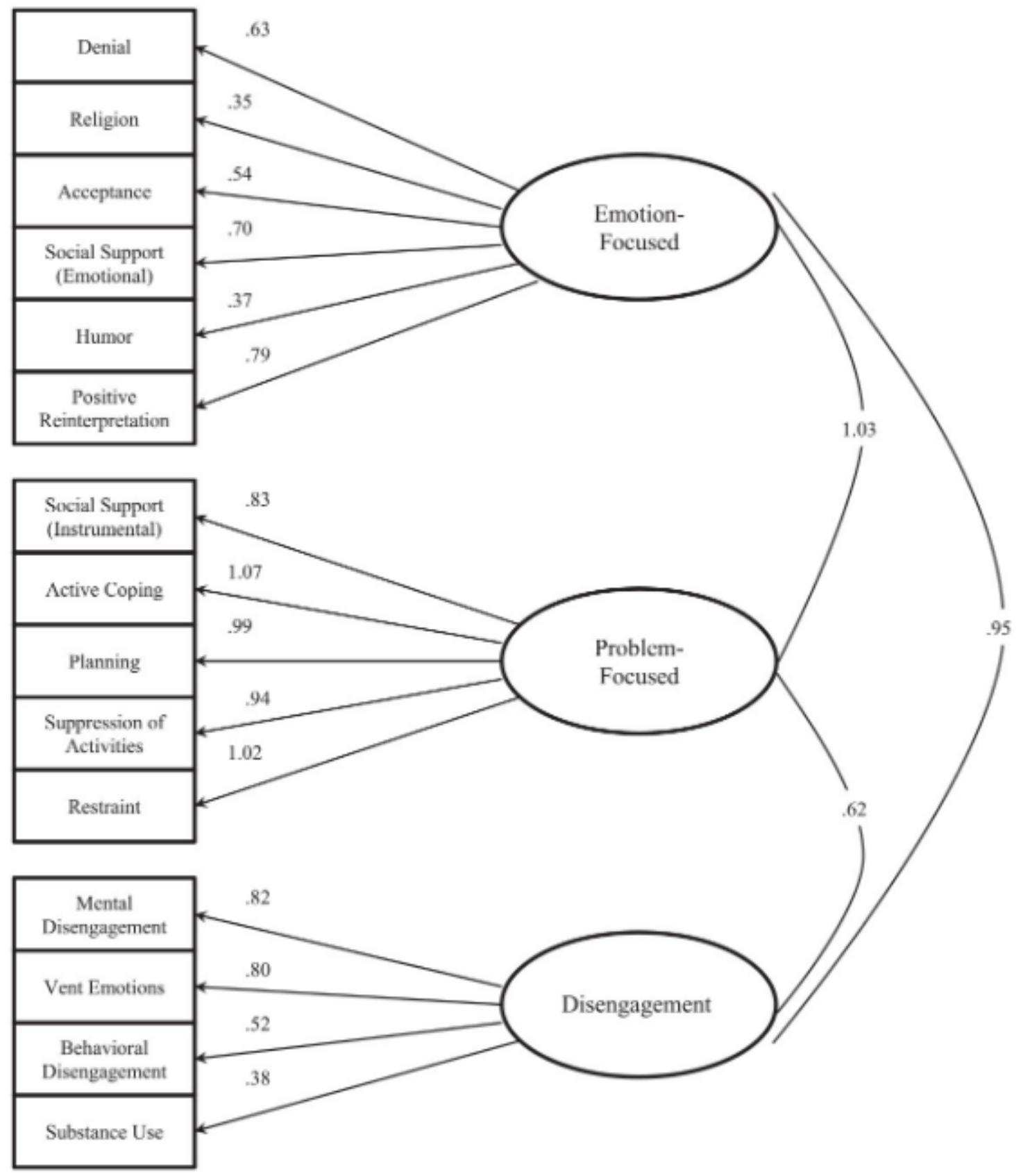

Figure 2. Alternate latent model for COPE subscales and factor loadings in current study. Note. Latent constructs are shown in ellipses and indicator variables are shown in rectangles. COPE $=$ Coping Orientation to Problems Experienced.

Qualitative Phase

Coping themes. Based on thematic analysis of the 14 interviews, five overarching major themes related to coping emerged (see Table 3 ). 
Table 3. Themes From Qualitative Interviews.

\begin{tabular}{|c|c|c|}
\hline Overarching themes & Participant examples & $\begin{array}{l}\text { COPE subscales with } \\
\text { some similarity }\end{array}$ \\
\hline Positive thinking and self-talk & $\begin{array}{l}\text { "stay positive," "just focus and overcome it," } \\
\text { "don't complain" and "say no to pressure" }\end{array}$ & Positive reinterpretation \\
\hline Relational coping & $\begin{array}{l}\text { "talk with sister who is close to me," "mom } \\
\text { gives advice and support," and "teachers or } \\
\text { friends who I trust" }\end{array}$ & Emotional social support \\
\hline Planning & $\begin{array}{l}\text { "stay organized," "practice for tests," “ask } \\
\text { my classmates," and "confront most } \\
\text { immediate problems" }\end{array}$ & $\begin{array}{l}\text { Planning; instrumental } \\
\text { social support }\end{array}$ \\
\hline Separate/disengage & $\begin{array}{l}\text { "running from problems," "keeping it in," } \\
\text { "ignore the person causing the problem," } \\
\text { and "block things out" }\end{array}$ & Mental disengagement \\
\hline $\begin{array}{l}\text { Behavioral coping (actions that help a } \\
\text { person calm down and recover balance) }\end{array}$ & $\begin{array}{l}\text { "walk outside," "listen to music," "splash } \\
\text { water on my face," "draw" and "relax" }\end{array}$ & None \\
\hline
\end{tabular}

Note. $\mathrm{COPE}=$ Coping Orientation to Problems Experienced.

Positive thinking/self-talk. The adolescent participants demonstrated their ability to cope by cognitive reframing with messages like "stay positive," and "just focus and overcome it." These positive messages were pervasive throughout all interviews, as demonstrated by the participant who said,

I always try to stay positive, if I, like, get sad or something, I mean, you start thinking things that are, that don't make sense or wanna harm yourself or things that you shouldn't be doing, and I'm, like, nah, I gotta stay positive.

This theme is similar to the COPE scale of positive reinterpretation, which includes items such as "I try to see it in a different light, to make it seem more positive." However, our participants also shared many examples of active self-talk, with a focus on using their cognitive reframing efforts to rise above the current stressors, not just to see them differently. Rather than simply looking for "something good in what is happening," as the COPE would frame it, these youth seemed to be acknowledging the difficult circumstances and stressors as real and then coaching themselves to respond with strength and positivity.

Relational coping. The overwhelming majority of the students interviewed said coping through relationships was crucial or essential to them. For example, one student responded,

Oh, well I think it's really important because you always need to have somebody to go talk to, somebody to tell your stuff to. Like I have a lot of friends that I can really trust, I can really talk to them about my problems or what I'm feeling. And that's the same way with my parents so, I guess it's really important to me because I can actually talk to other people and they understand.

Another student discussed an incident of bullying at school, and how he or she used a trusted friend to help determine the best response, which was to include a teacher in the situation.

Ahh, when I got to the school I was a pretty shy person and there was someone making fun of me. Umm, I didn't really wanna tell the teacher because it's like they'll say I'm a 
tattle teller and then I don't really like that. But my friend told me that to tell on the teacher 'cause it's not tattle telling it's like reporting 'cause it's something serious.

This focus on relationships as critically important would be expected in terms of familism and collective cultural values in Latinx immigrant families. Students noted that they talked with "teachers or peers who are trusted," and with specific family members about their concerns. This seems to be built on an important foundation of trust and connection, and central to our participants' coping strategies. In comparison, the emotional social support subscale in the COPE is represented by items such as "I discuss my feelings with someone." The relational coping theme appeared more central and essential in the current interviews.

Plan. Students indicated that they could cope with challenges at school by staying organized, practicing for upcoming tests, focusing on the work they needed to get done, and confronting the most immediate problems. One student provided an example of how being more planful could help them at school, such as "Right now I'm taking some higher level classes, so sometimes I don't understand the topic, so I either ask my brother or ask my classmates." Students also considered long-range educational planning, as reflected in the following quote.

Well something difficult like, I've been thinking about my future college and what I want to study and that's a problem that I've had lately . . . I just do research and I just talk to classmates or my mom about it.

A different example of organizing or planning in order to cope with a situation at home was as follows:

Mm, so with the thing about my dad and me and him wanting me to help around the house more, I've tried to kind of like write myself little notes in my phone sometimes, some reminders telling me to do this and to do that. So that way I can remember to help out more and that's something that's helped me out a lot, is being more organized.

Planning is also a COPE subscale, as illustrated by the item "I try to come up with a strategy about what to do." Our participants mobilized their support networks (going back to the central role of relationships in coping) in order to generate more ideas about planning behaviors at school. In this way, instrumental social support (a COPE subscale) was needed to assist with the planning function. Students indicated that friends could help them with some academic tasks at school, but students in immigrant families may be on their own in terms of people they can turn to for strategies that are grounded in an understanding of the workings of U.S. schools.

Separate/disengage. Participants said they would cope with stressors at times by "running from the problems," or "blocking things out." Some participants did acknowledge that this strategy meant they were avoiding the problem for a time or procrastinating to delay having to deal with it. This seems to overlap with some of the items in the mental disengagement subscale in the COPE, such as "I go to movies or watch TV to think about it less," or "I daydream about things other than this." In this sample, blocking out the problems or stressors seemed to go hand in hand with the theme of positive thinking, such as in the following quote: 
Umm yea like I guess it would if I have a problem I try not to think about it too much. I'm just like, tell myself, like you know, just calm down and just finish what you're doing, you'll deal with it like you know, later. Or it won't bother me 'cause I try not, I try not to always get mad at the situation, I guess you could say. I'm not sure.

Behavioral coping. Participants described many strategies for calming back down or recovering their balance and positive affect in times of stress, including walking outside, listening to music, drawing, splashing water on their faces, praying, watching TV, playing sports, or just calming down alone in their rooms. These behaviors were present-focused in a way that was either active or contemplative. One student listed strategies for dealing with a stressful day:

Or I think of like a calm time, a calm time would be like usually what I do every day. Umm I draw or listen to music because that really helps me calm down . . . all I want to do in life is sit down and just draw because that's like the calmest thing for me. Like either I always have my cat there and I'll be happy because I love my kitties and drawing that usually calms me down whenever I get home from a long tough day at school.

These strategies are different from the active coping items on the COPE, which emphasize taking direct action specific to the stressor, concentrating one's efforts on solving the problem, and doing what needs to be done, one step at a time. These behavioral strategies are also not the same as the mental disengagement items on the COPE, which might include sleeping more or distracting oneself. These participants seem to be indicating that they are purposefully calming themselves as they acknowledge the stressor and not with the primary purpose to avoid the stressor. This theme is reminiscent of the shift and persist strategies (Chen \& Miller, 2012) in the sense of reframing and refocusing attention on something that brings meaning or calm. Finally, behavioral coping in this sample included a small set of behaviors that could be connected to religious coping (praying), but not as a predominant strategy.

We saw little to no evidence in the interviews of adolescents endorsing coping through substance abuse, venting, denial, restraint, behavioral disengagement, and humor as defined in the COPE. That does not mean that those coping styles may not be present, but that they were not noted in the interviews. In particular, giving up (behavioral disengagement in the COPE) was not an idea that was endorsed at all in the current study. Indeed, much of the positive self-talk that we heard in the interviews was directly contrary to the idea of giving up.

Culturally based coping. In addition to the five qualitative themes, the interviews illuminated some of the Latinx-specific cultural values that have been described in the literature review but are not part of traditional coping measures. This culturally based content did not emerge as a theme in the semantic coding strategy that looked for specific coping strategies, but was seen as part of the context underlying many of the examples of coping that were given. For example, students described their ability to access help and support for coping using two languages or two cultural styles. In the words of one participant,

It's kind of like my parents, they listen to, they get advice from their friends, and if I couldn't understand Spanish, I couldn't get that information that they have. So, it's kind 
of like the Spanish speaking culture has different advice to offer than the English culture. So I can, like, combine those two, and it helps me deal with my own problems.

Participants also described the cultural clashes that can occur between their different worlds and how they must adjust their approach to each setting. In one participant's words, "Like, I would change the way I think a little, to what school is, 'cause school is a different thing from what home is and like, it's really different, like two worlds that are way apart." These different roles and expectations for children in immigrant families have been identified in previous literature, but not tied directly to the idea of coping strategies and how youth can manage differing expectations at school and home. Another participant thoughtfully noted,

It's just that sometimes me and my dad have different ideas of what my role should be at home. Because I spend a lot of time at school with clubs and stuff and he says that is good for me and all, but he wants me to be home a lot more too because he says, and it's true, that I am not really home too much. I really don't help clean up around here too much. I know that on my part I try all the time to think about what he says, and try to do stuff at home too, and spend less time at school, maybe.

Finally, some students also said that they "remember who I am or where I come from" as a reference to drawing on cultural pride or traditions for strength in coping. Students also underscored in many ways the important role of family and of taking their obligations to contribute to their families very seriously (Kiang \& Fuligni, 2010). A sense of duty and connection to family members and willingness to undertake those tasks was suffused throughout.

Integrating Mixed-Methods Findings

The researchers reflected on the nuances in qualitative data, which helped provide additional perspective on the three factors that arose in the EFA labeled "Purposeful cognitive/behavioral engagement," "Support seeking," and "Separation/disengagement." There are differences with the three factors that are present in the COPE measure (per Carver et al., 1989) and the current study. The COPE Factor 1 (problem-focused coping) has planning, suppression of competing activities, restraints, and active coping in common with our Factor 1. However, our factor (purposeful cognitive/behavioral engagement) also includes positive reinterpretation and acceptance along with humor and religion. Factor 2 in the COPE (emotion-focused coping) only has one variable in common with our Factor 2 (support seeking), which is emotional support. For our participants, instrumental social support and venting also loaded onto Factor 2. Finally, the COPE Factor 3 (disengagement) and our Factor 3 (separation/disengagement) have substance abuse, behavioral disengagement, and mental disengagement in common. Denial also loaded onto our Factor 3, but not in the COPE model.

\section{Discussion}

The purpose of the current study was to explore the fit of the traditional COPE model (Carver et al., 1989) with data gathered from youth living in Latinx immigrant families, using cultural relevance as a critical lens. We addressed a gap in the literature by drawing on the strengths of both qualitative and quantitative data to explore unique culturally informed coping strategies in 
this population. The qualitative themes added nuance to the three factors identified in the EFA, which were not exactly the same as the three factors from the original COPE model. One way to understand this potential synergy in our mixed-methods data is to consider the themes of positive thinking/self-talk and behavioral coping as connected to Factor 1 (purposeful cognitive/behavioral engagement), the theme of relational coping as deepening our understanding of Factor 2 (support seeking), and the theme of separate/disengage as consistent with Factor 3 (separation/disengagement). It is less clear how the planning theme might fit within the proposed alternate approach to coping. Together, the mixed-methods findings emphasize (a) the importance of trusted relationships and interdependence to many of the coping strategies implemented by the Latinx youth, (b) the way that positive self-talk helped youth shift away from negative thinking or affect and find ways to be resilient in the face of difficulty, and (c) the behaviors that helped participants ground themselves in the present moment in the face of stressors. Family was mentioned throughout the discussions of coping strategies, beyond what might be thought of simply as "emotional support seeking."

As expected, the CFA indicated that the traditional COPE model was not a good fit for this population. If we understand coping as the interaction of an individual with his or her environment, then the unique context of youth living in immigrant families may present both stressors that are culturally distinct (e.g., acculturative stress and racial-ethnic discrimination) and strengths and strategies that are culturally based (e.g., collectivism, familism, ethnic identity, bilingualism, and biculturalism). Seen from this ecological vantage point, it makes sense that coping in Latinx youth may not parallel the traditional models. Indeed, our findings are in line with the few studies found in the literature that regard coping in collectivist cultural traditions (Kuo, 2013; Moore \& Constantine, 2005) with an underlying theme of interdependence and relational coping running through the results. For example, even an activity like planning, which has tended to be considered in a predominantly cognitive way in prior coping models, was enacted by our participants in a communal and interdependent way. Thus, our contribution is to suggest one way to broaden the view of coping in a Latinx sample, question cultural assumptions of the traditional models, and to encourage further work along these lines.

In the EFA, some of the ways that coping may be structured differently were suggested. For example, the items that would have been considered as emotion-focused coping by Carver et al. (1989) were actually distributed across all three of the factors identified in the current study. Although we cannot definitively identify the reason, we can make suggestions that would be supported by the literature on Latinx immigrant families. If we consider some of the core values that help frame the worldview of Latinx immigrant families, we return to familism, collectivism, and ethnic identity (Stein et al., 2014). Thus, problem-focused coping likely would not be an individualized endeavor for our participants, but would be connected to key relationships within the family and a core sense of existing within that web of relationships. Engaged coping through positive reinterpretation could be couched in the wisdom and affirmations passed down from family members or balanced by a sense of emotional acceptance that some things are beyond our ability to control.

Indeed, some of those interpretations were mirrored in the qualitative findings, where students referred to their family members as essential parts of their support system and talked about the ways they would gain perspective, assistance, comfort, and encouragement within their circle of 
trusted peers and adults. Relational coping strategies were prevalent throughout the interview data, just as emotional coping was present across all three factors in the EFA. The central importance of connection to family for buffering against stress has been highlighted by other researchers, such as one study where higher levels of familism were associated with more engagement coping for Latinx youth in the face of stressors (Santiago et al., 2016), Thus, future work on coping with Latinx adolescents should use measures that incorporate relational and collectivistic coping at a minimum (Moore \& Constantine, 2005) as those themes were pervasive in the qualitative interviews in this study.

Relational coping for Latinx youth may be particularly important in the context of discrimination, bullying, and acculturative stress. As in other studies (Brittian et al., 2013; McDermott, Umaña-Taylor, \& Zeiders, 2019; Sanchez et al., 2018), incidents of racialethnic bias were noted as relevant stressors for this population. Students did mention bias and bullying in the interviews, usually when asked to think about a school-based situation that had caused them stress. Thus, one limitation of generalized quantitative coping measures could be their lower relevance to the life experiences of disenfranchised youth and families. If coping is indeed situation and context specific, then more generalized coping models should be closely examined to see whether they are adequate for the specific task of confronting discrimination. Some previous studies that have examined coping responses to discrimination (McDermott, Umaña-Taylor, \& Martinez-Fuentes, 2019) have utilized a specific measure (e.g., discrimination coping strategies scale) and not a generalized coping scale. Racial/ethnic discrimination cannot be ignored in studies of coping for Latinx youth.

The racial-ethnic context of this particular study in an emerging immigrant community, and indeed the anti-immigrant sentiment that is present in our current public discourse, was inescapable. The literature related to coping in marginalized populations has differed on the value of engaged coping strategies (proactively addressing a stressor) versus disengaged coping strategies when addressing discrimination as a stressor. Some studies have found that the disengaged coping styles (including forbearance) have been associated with more negative outcomes in the long term (lower mental health and lower college persistence attitudes) (Hernández \& Villodas, 2019; Sanchez et al., 2018). McDermott, Umaña-Taylor, \& Zeiders (2019) found that ignoring a discriminatory event could lead to greater internalizing symptoms, but this form of disengaged coping did help youth in school settings by allowing them to focus on their academic work. For a student facing limited financial resources, discrimination, or fears of family separation due to deportation, coping by denying those realities or giving up may provide an initial buffering effect (McDermott, Umaña-Taylor, \& Zeiders, 2019) but may not be helpful as long-term strategies. Future studies with Latinx youth should examine the question of when to show forbearance or disengagement strategies and when to actively engage or seek support in the face of discrimination.

The disengaged coping style did load onto one of the factors in our EFA using the quantitative data but was not as prominent in real-life coping narratives in the qualitative data. Participants provided many examples of engaged coping styles wherein students sought to connect with their primary support systems or engage in positive self-talk and planning, but the interviews did not provide examples of denial, behavioral disengagement, use of alcohol, and restraint as strategies. However, shift and persist theory suggests that disengaging (i.e., shifting) from uncontrollable 
stressors while focusing on things that provide youth with purpose, meaning, and hope is an effective coping strategy to combat the negative effects of socioeconomic stress for youth in poverty (Chen \& Miller, 2012). This specific type of coping was suggested in the positive thinking/self-talk and behavioral coping themes in the qualitative study. For Latinx youth, familism and a relational orientation may be relevant for both the shift and the persist phases as their sense of meaning and purpose may be specifically tied to their familism values and behaviors (Kiang \& Fuligni, 2010). Given that shift and persist has been predictive of better health outcomes for marginalized youth, future work should consider how shift and persist may function for Latinx immigrants (Christophe et al., 2019). Our findings underscore the value in considering alternate approaches to coping for collectivist cultural traditions.

In addition to bringing nuance to the discussion of whether traditional coping models truly fit this population, the qualitative data also shed light on culturally based coping. While participants didn't specifically mention culturally based coping strategies in response to the interview questions, the presence of language and culture undergirded much of their interviews. For example, students who maintained fluency in both Spanish and English noted that they were then able to access advice and strategies from their parents at home as well as from teachers and peers at school. Thus, bilingualism was like a key that opened the doors for multiple perspectives on coping and allowed students to select strategies that were effective, given the setting they were in or the type of stressor they were encountering. Moreover, the literature has documented the positive influence of cultural factors, such as a strong racial-ethnic identity and endorsement of familism for Latinx youth well-being (Stein et al., 2014). By considering cultural factors as embedded across youth's daily proximal processes (Vélez-Agosto et al., 2017), such as the use of relationships in coping behaviors when engaging with daily stressors, we can begin to hypothesize that cultural values like familism may be related to the increased importance placed on relational coping and positive self-talk.

\section{Limitations and Future Research}

One important limitation of the current study is that we were not able to enter each of the 60 COPE items individually in the EFA because the sample size did not support it. Additional work with a larger sample could allow the items to move independently within the factor analysis, but we were confined to working within the existing 15 subscales of the original COPE. In addition, when the COPE data were collected, we did not ask participants to indicate what stressful situation they were thinking of specifically but only whether it was home or school context. Especially for youth in immigrant households, it would be important to understand the specific stressors that were testing their coping strategies. There were oblique mentions of the current political climate in the qualitative interviews, but that was not a focus of the investigation. In terms of context, it would also be beneficial to follow up on the bicultural or bilingual aspects of coping with stressors in the Spanish-speaking world of home and the English-speaking world of school. Another limitation in terms of developmental stages is that the quantitative data were drawn from participants with an average age of 12.9 years, while the qualitative interview participants had an average age of 15.7 years. The purpose of the current study was not to elaborate on developmental differences in coping but age can be an important aspect for future research. Finally, the EFA in the current study showed one alternative way to consider coping in this population, but we encourage other researchers to test such a model more rigorously to 
confirm it and to consider including related variables (familism, ethnic identity, and collectivism).

Implications and Conclusion

Although this study was exploratory in nature, it provides a starting place for thinking about ways to support coping in Latinx youth from immigrant families. For example, this study underscores the importance of considering how culture might influence our perception of stressors, the way we make meaning from those experiences, selecting coping strategies, and identifying resources we can use to strengthen our responses (Kuo, 2013). Do existing coping models address how marginalized youth encountering racial/ethnic bias or discrimination deal with these pervasive stressors? Do the set of strategies known as "shift and persist" offer a better foundation for dealing with uncontrollable stressors like poverty and racism/xenophobia (Chen \& Miller, 2012)? These are important questions with direct implications on how to best help youth find strategies to deal with their daily stressors.

In addition, the importance of collectivism and key relationships to coping strategies for youth in immigrant families is an insight worth pursuing in intervention work. The resilience described as positive self-talk and behavioral coping could be important for clinicians, teachers, and parents to foment as these types of strategies may be helpful for youth facing uncontrollable stressors. Differences in the types of stressors faced, the cultural strengths, identities, and values available, and the amount of support available in the nearby environment could all impact which coping strategies might be best (Brittian et al., 2013; Hernández \& Villodas, 2019; Sanchez et al., 2018), which has implications for how we socialize children to build their coping skills.

\section{Declaration of Conflicting Interests}

The author(s) declared no potential conflicts of interest with respect to the research, authorship, and/or publication of this article.

\section{Funding}

The author(s) received no financial support for the research, authorship, and/or publication of this article.

\section{ORCID iD}

Laura M. Gonzalez (iD https://orcid.org/0000-0003-4738-1288

\section{References}

Aldridge, A. A., Roesch, S. C. (2008). Developing coping typologies of minority adolescents: A latent profile analysis. Journal of Adolescence, 31, 499-517.

Asparouhov, T., Muthén, B. (2009). Exploratory structural equation modeling. Structural Equation Modeling, 16, 397-438. https://doi.org/10.1080/10705510903008204

Bentler, P. M., Bonett, D. G. (1980). Significance tests and goodness of fit in the analysis of covariance structures. Psychological Bulletin, 24, 445-455. 
Braun, V., Clarke, V. (2006). Using thematic analysis in psychology. Qualitative Research in Psychology, 3, 77-101.

Brietzke, M., Perreira, K. (2017). Stress and coping: Latino youth coming of age in a new Latino destination. Journal of Adolescent Research, 32, 407-432.

Brittian, A. S., Toomey, R. B., Gonzales, N. A., Dumka, L. E. (2013). Perceived discrimination, coping strategies, and Mexican origin adolescents' internalizing and externalizing behaviors: Examining the moderating role of gender and cultural orientation. Applied Developmental Science, 17, 4-19.

Carver, C. S., Scheier, M. F., Weintraub, J. K. (1989). Assessing coping strategies: A theoretically based approach. Journal of Personality and Social Psychology, 56, 267-283.

Chen, E., Miller, G. E. (2012). "Shift-and-persist" strategies: Why low socioeconomic status isn't always bad for health. Perspectives on Psychological Science, 7, 135-158. https://doi.org/10.1177/1745691612436694

Christophe, N. K., Stein, G. L., Romero, M. Y., Chan, M., Jensen, M., Gonzalez, L. M., Kiang, LAuthors. (2019). Coping and culture: The protective effects of shift-and-persist and ethnicracial identity on depressive symptoms in Latinx youth. Journal of Youth and Adolescence, 48, 1592-1604.

Connor-Smith, J. K., Compas, B. E., Wadsworth, M. E., Thomsen, A. H., Saltzman, H. (2000). Responses to stress in adolescence: Measurement of coping and involuntary stress responses. Journal of Consulting and Clinical Psychology, 68, 976-992.

Gloria, A. M., Castellanos, J., Delgado-Guerrero, M., Salazar, A. C., Nieves, C. M., Mejia, A., Martinez, V. L. (2017). El ojo en la meta: Latino male undergraduates' coping processes. Journal of Diversity in Higher Education, 10, 11-24.

Greer, T. M. (2007). Measuring coping strategies among African Americans: An exploration of the latent structure of the COPE Inventory. Journal of Black Psychology, 33, 260-277.

Hanson, W. E., Creswell, J. W., Clark, V. L. P., Petska, K. S., Creswell, J. D. (2005). Mixed methods research designs in counseling psychology. Journal of Counseling Psychology, 52, 224235.

Hernández, R. J., Villodas, M. (2019). Collectivistic coping responses to racial microaggressions associated with Latina/o college persistence attitudes. Journal of Latinx Psychology, 7, 76-90. https://doi-org.libproxy.uncg.edu/10.1037/lat0000107

Hu, L., Bentler, P. M. (1999). Cutoff criteria for fit indexes in covariance structure analysis: Conventional criteria versus new alternatives. Structural Equation Modeling, 6, 1-55. https://doi.org/10.1080/10705519909540118 
Kiang, L., Fuligni, A. J. (2010). Meaning in life as a mediator of ethnic identity and adjustment among adolescents from Latin, Asian, and European American backgrounds. Journal of Youth and Adolescence, 39, 1253-1264.

Kuo, B. C. (2011). Culture's consequences on coping: Theories, evidences, and dimensionalities. Journal of Cross-Cultural Psychology, 42, 1084-1100.

Kuo, B. C. (2013). Collectivism and coping: Current theories, evidence, and measurements of collective coping. International Journal of Psychology, 48, 374-388.

Marsh, H. W., Hau, K. T., Wen, Z. (2004). In search of golden rules: Comment on hypothesistesting approaches to setting cutoff values for fit indexes and dangers in overgeneralizing Hu and Bentler's (1999) findings. Structural Equation Modeling, 11, 320-341.

https://doi.org/10.1207/s15328007sem1103_2

McDermott, E. R., Umaña-Taylor, A. J., Martinez-Fuentes, S. (2019). Measuring Latino adolescents' coping with ethnic-racial discrimination. Journal of Psychoeducational Assessment, $37,730-742$.

McDermott, E. R., Umaña-Taylor, A. J., Zeiders, K. H. (2019). Profiles of coping with ethnicracial discrimination and Latina/o adolescents' adjustment. Journal of Youth and Adolescence, 48, 908-923.

Moore, J. L., Constantine, M. G. (2005). Development and initial validation of the collectivistic coping styles measure with African, Asian, and Latin American international students. Journal of Mental Health Counseling, 27, 329-347.

Sanchez, D., Smith, L. V., Adams, W. (2018). The relationships among perceived discrimination, Marianismo gender role attitudes, racial-ethnic socialization, coping styles, and mental health outcomes in Latina college students. Journal of Latina/o Psychology, 6, 1-15. https://doi.org/10.1037/lat0000077

Santiago, C. D., Torres, S. A., Brewer, S. K., Fuller, A. K., Lennon, J. M. (2016). The effect of cultural factors on daily coping and involuntary responses to stress among low-income Latino adolescents. Journal of Community Psychology, 44, 872-887.

Skinner, E. A., Zimmer-Gembeck, M. J. (2007). The development of coping. Annual Review of Psychology, 58, 119-144.

Stafford, A. M., Bigatti, S. M., Draucker, C. B. (2019). Cultural stressors experienced by young Latinas with depressive symptoms living in a tumultuous sociopolitical climate in the United States. Archives of Psychiatric Nursing, 33, 36-42.

Stein, G. L., Cupito, A. M., Mendez, J. L., Prandoni, J., Huq, N., Westerberg, D. (2014). Familism through a developmental lens. Journal of Latina/o Psychology, 2, 224-250. 
Stein, G. L., Gonzalez, L. M., Huq, NAuthors. (2012). Cultural stressors and the hopelessness model of depression in Latino adolescents. Journal of Youth and Adolescence, 41, 1339-1349.

Taylor, Z. E., Widaman, K. F., Robins, R. W. (2018). Longitudinal relations of economic hardship and effortful control to active coping in Latino youth. Journal of Research on Adolescence, 28, 396-411.

Umaña-Taylor, A. J., Vargas-Chanes, D., Garcia, C. D., Gonzales-Backen, M. (2008). A longitudinal examination of Latino adolescents' ethnic identity, coping with discrimination, and self-esteem. The Journal of Early Adolescence, 28, 16-50.

Vélez-Agosto, N. M., Soto-Crespo, J. G., Vizcarrondo-Oppenheimer, M., Vega-Molina, S., García Coll, C. (2017). Bronfenbrenner's bioecological theory revision: Moving culture from the macro into the micro. Perspectives on Psychological Science, 12, 900-910.

Yeh, C. J., Arora, A. K., Wu, K. A. (2006). A new theoretical model of collectivistic coping. In Wong, P. T. P., Wong, L. C. J. (Eds.), Handbook of multicultural perspectives on stress and coping (pp. 55-72). Springer.

Laura M. Gonzalez is an associate professor of higher education in the Department of Teacher Education/Higher Education at the University of North Carolina at Greensboro. Her doctorate in counselor education was from North Carolina State University and her current research interests are related to college access for youth from Latinx immigrant families.

Yesenia Mejia is a doctoral student in clinical psychology at the University of North Carolina at Greensboro. Her research interests are in using developmental cascade models to understand links between depressive symptoms and academic functioning in Latinx adolescents.

Andrea Kulish is a doctoral student in clinical psychology at the University of North Carolina at Greensboro. Her research focuses on parent-child communication about emotion in Latinx families.

Gabriela Livas Stein is an associate professor in clinical psychology at the University of North Carolina at Greensboro. She received her PhD from the University of North Carolina at Chapel Hill. Her research focuses on culturally protective processes in Latinx families.

Lisa Kiang is a professor of psychology at Wake Forest University. She received her $\mathrm{PhD}$ from the University of Denver. Her area of research is in cultural identity and social relationships, with an emphasis on positive well-being in ethnically diverse adolescents.

Deja Fitzgerald is an area coordinator at Reed College and a research associate for Martinez Consulting Firm. Her masters of education was from University of North Carolina at Greensboro, and her current research interests are diversity in industrial-organizational psychology and employee selection for high-stress, high-reliability, and high-risk organizations. 
Alyson Cavanaugh is a research scientist at Frank Porter Graham Child Development Institute at the University of North Carolina at Chapel Hill. Her major research interests include educational, psychological, and cultural experiences of ethnic minority youth. 